Research Article

Open Access

Olusegun Emmanuel Afolabi

\title{
Clinical Significance: a Therapeutic Approach Topsychological Assessment in Treatment Planning
}

DOI 10.1515/sigtem-2016-0003

\begin{abstract}
Psychological assessment has long been reported as a key component of clinical psychology. This paper examines the complexities surrounding the clinical significance of therapeutic approach to treatment planning. To achieve this objective, the paper searched and used the PsycINFO and PubMed databases and the reference sections of chapters and journal articles to analysed, 1) a strong basis for the usage of therapeutic approach to psychological assessment in treatment plans, 2) explained the conceptual meaning of clinical significant change in therapeutic assessment, 3) answered some of the questions regarding practicability and the clinical significance of therapeutic approach to treatment plans, particularly during or before treatment, 4) linked therapeutic assessment to change in clients' clinical impression, functioning and therapeutic needs 5) analysed the empirically documenting clinically significant change in therapeutic assessment. Finally, the study suggested that though therapeutic assessment is not sufficient for the systematic study of psychotherapy outcome and process, it is still consistent with both the layman and professional expectations regarding treatment outcome and also provides a precise method for classifying clients as 'changed' or 'unchanged' on the basis of clinical significance criteria.
\end{abstract}

Keywords: therapeutic approach, psychological assessment, clinical significant change, treatment outcome.

\section{Introduction}

Psychological assessment is to some degree of a crossroads. Though research has long suggested that psychological assessment should move from psychometrics and scale development to a pragmatic assessment (Meyer et al., 2001), raising this issue among psychologists reliably revealed robust and conflicting opinions. While evidence shows that psychological assessment covers a nontrivial part of clinical activities (Norcross, Karpiak, \& Santoro, 2005), drops in graduate training in assessment (e.g., Curry \& Hanson, 2010) and changed reimbursement from managed mental health care (e.g., Eisman et al., 2000) consistently influenced its usage in clinical practice. However, despite these challenges, the use of the traditional method of assessment for treatment continues to be a major discourse among researchers and practitioners to date. This method was limited in two respects; first, psychological assessments offered no statistical information on the inconsistency in client's response to treatment despite the relevance of the information on variability of treatment outcome. Second, the effect of psychological assessment is less significant on clinical outcome.

Though much has been done in the past to promote clinical significant change in therapeutic assessment, their outcomes are yet to be proved and analysed systematically (Finn, Fischer, \& Handler, 2012). The

*Corresponding author: Olusegun Emmanuel Afolabi: Department of Educational Foundation, University of Botswana, P O Box 501757, Gaborone, Botswana, E-mail: afo13@yahoo.com 
clinical significance of therapeutic assessment to treatment refers to its proficiency to attain the values of competence set by clients, clinicians, and scholars. While there was little agreement about the standards, the available research confirmed many propositions on the issue. Some of this include: a high proportion of clients improving; a level of adjustment that is identifiable by peers and significant others (Wolf, 1978); removal of the current problem (Kazdin \& Wilson, 1978); normative levels of effectiveness by the end of therapy (Nietzel\& Trull, 1988); or changes that meaningfully lessen the risk from other health problems to mention a few. This shift in psychological assessment, particularly as it related to envision and collective oriented therapy has infused a new life into the debate on clinical significant change in clients' treatment outcome. The measures and models that come from this paradigm shift were categorised as therapeutic assessment (Finn, Fischer, \& Handler, 2012). These were shared and documented by various scholars (e.g., Meyer et al., 2001) as a concept that prompts therapeutic change in treatment. However, despite this development, clinicians still found it hard to agree on whether a change in clients' condition can be attributed to the assessment or not (Jacobson, Follette, \& Revenstorf, 1984).

\section{The Aim of the Study}

This article brings together several empirical findings that support the clinical significance of therapeutic assessment in treatment planning. It also aimed at answering some of the questions regarding the clinical significant change during or before treatment. The paper linked therapeutic assessment to intervention process that identified, described and managed clients' functioning, clinical impression and therapeutic needs. To achieve these objectives, the paper empirically outlined the following; 1) a strong basis for the usage of therapeutic approach to psychological assessment in treatment plans, 2) examined the conceptual meaning of clinical significant change in therapeutic assessment, 3) used initial theory to explain the therapeutic mechanisms of change in psychological assessment, and analysed the common deficiency identified in treatment validity, and finally, 4) gathered empirical evidence to support the clinical significant change in therapeutic assessment in clinical practice.

\section{Materials and Methods}

This paper analysed and reviewed empirical literature that highlights the clinical significance of therapeutic approach to treatment planning. It also addressed the complexities, practicability and acceptability of therapeutic approach to psychological assessment. The study collated and reviewed relevant articles, books, journals, and meta-analysis on clinical significance of therapeutic approach to psychological assessment. Both the ERIC and PSYCHLIT databases were searched using the following key words: therapeutic approach, clinical significant change, treatment outcome, treatment planning and psychological assessment. This procedure initially reported about 1650 articles, journals, technical reports, paper presentation, case studies and book chapters covering more than 28-year period. Based on the abstracts retrieved from this initial 1650 plus articles and publications, the search was lessened to a relatively few hundred of studies that are pertinent, current and relevant to the theme of this paper. The contents of the remaining several hundred articles cum journals were further scrutinised and only those that reported empirical findings were kept aside and used, while others were left out for further consideration. The process shows that only a few studies documented empirical findings on clinical significance of therapeutic approach to psychological assessment in clinical practice. To verify references, manual searches of relevant journals and articles related to the paper are performed.

\section{Results}

Therapeutic assessment is a short-term highly organised hypothetically and scientifically grounded method of psychological assessment. This method though arguable, was established by Stephen Finn and 
his professional colleagues and was significantly swayed by the humanistic school and self-psychology. Historically, therapeutic intervention was linked to the work of the humanistic crusade of the 1950s and 1960s. Though many humanistic-oriented clinicians (Rogers, 1951) were strongly against the use of psychological assessment, its experimental utility has continued to grow over the years and has been crucial for effective treatment planning in clinical practice. The period between 1960s and1970s also saw scholars such as Goldman (1972) describe assessment and treatment as 'a failure marriage' (p. 213). Besides, some of these scholars also had a long-held conviction that professional involvement of clients in assessment was injurious and unhealthy (e.g., Eisman et al., 2000).

Of most interest among this view is the way they put a diverse twist on psychological assessment and its response method. For instance, some psychologist looked at psychological assessment as a therapeutic interpersonal knowledge rather than a clean reductionist practice (Riddle, Byers, \& Grimesey, 2002). Yet, despite the avalanche of criticism, therapeutic assessment continues to spread and covers important and specific areas such as (a) assisting service users to develop questions they need to solve through the assessment and testing, (b) gathering contextual evidence associated with their problems, (c) using previous assessment (d) engaging clients in partnership and making logic of the results, and last but not the least, provide immediate response to clients' early questions (Finn, 2007). Up till now, the question remains, thus this approach really therapeutic enough to bring appropriate change in clients' condition. So far, this continues to face empirical test and till now, no agreement was made on the issue.

\section{Theoretical basis for clinical significant change in therapeutic assessment}

The use of the traditional evaluation for treatment plan has been long criticised. In fact, the process comes with some limitation, particularly on measuring change in clients' condition. Both the applied research and clinical practice confirmed that assessing change that clients experience as a result of their treatment is a common practice in therapy. To quantify the change in clients' condition in order to correctly evaluate the significance of the treatment is very cumbersome, to say the least challenging. Though the null hypothesis significance testing provides professionals with valuable information during treatment, it failed to highlight the significance of the estimated effect because it partly relies on sample size (Thompson, 2002). While the measures of outcome size has been used to cover this constraint, the fact remains that the more the observed outcome is, the more likely it relates to a clinically meaningful change. While it was reported that these types of measures hinge on the variability of the analysed scores, it was also agreed that a large outcome does not ineludibly link with an important effect (Jacobson, Roberts, Berns \& McGlinchey, 1999; Kazdin, 2001). Though the null hypothesis significance testing and the measures of outcome size are used to analyse the difference between group averages, they failed to detect the individual change in treatment outcome. Moreover, the process also failed to stipulate whether or not there is a particular change in clients' condition and at what percentage (Jacobson \& Truax, 1991). Therefore, the limitations of statistical tools in assessing intervention outcome prompted the change from statistical to clinical significance (see Ogles, Lunnen, \& Bonesteel, 2001).

\section{Conceptual meaning of clinically significant change in therapeutic assessment}

In recent time, the interest in clinical significance has grown not only in psychological research, but also in other health-related research measuring the quality of life and patient-reported outcomes (see Crosby, Kolotkin, \& Williams, 2003). In therapeutic assessment, the degree of change is the most remarkable characteristic of the meaning of clinical significance of therapeutic assessment. Earlier research on therapeutic assessment points toward a rather large, dependable change in symptoms and coming back to normative levels as primary manifestations of clinical significance. Clinical significance in a normal sense refers to as getting back to normal functioning. In the field of psychology, for example, clinical significance is typically related to the expression clinically meaningful change (CMC; see, e.g., Jacobson et al., 1984). In this perspective, it means 
'the practical value or significance of the outcome of an intervention, that is, whether the treatment makes a genuine change in clients life or to significant others in their life' (Kazdin, 2001, p. 455). Specifically, clinically significant change occurs when there is a big change in symptoms, an average change in symptoms and no change in symptoms. Though many contradictory claims had been made regarding the relation of the volume of change and clinical significance, the issue is still open to debate.

The idea that any volume of change in therapeutic assessment might be clinically significant is not casuistry, but rather expresses that clinical significance can and does mean many things, and these differ based on the kind of problems and the objectives of treatment. Though this might be opposite in some illnesses and may be too plain a criterion, however, it was founded on the postulation that clients came for treatment with a belief to get better or improve their condition. Even in situations where the criterion is too severe, the body of research, along with users of psychological services, hope to see how frequently can a client attain normal functioning after treatment. Although there were many other thoughts to this: For example, the degree of change in a given client must be statistically dependable, that is, it must get beyond the level of what could be sensibly ascribed to a mere chance or measurement error. Also, the final outcome in a given client is ascribed as a double criterion for clinically significant change: (a) the extent of the change has to be statistically dependable and (b) at the time of discharge, clients' condition must be in a level that makes them indistinguishable or at equilibrium with well-functioning people. However, if clients show a sign that was statistically reliable, but the treatment outcome to a certain degree was dysfunctional, then the client is considered as 'better, but not recuperated'. Moreover, if a client is finally found in a functional range at the end of the treatment and the extent of the adjustment is not statistically dependable, then the process cannot be justified whether or not the variation found in the treatment outcome is clinically significant. Finally, if the degree of change is statistically consistent and the client found himself within the usual limits on the variable of interest, the client can be adjudged to have 'recovered'. This metric offers the clinicians the opportunity to analyse how often the statistically significant decline can occur in therapeutic assessment by recognised clients who displayed a statistically dependable change opposite to those that suggested an improvement.

\section{General considerations that determined the clinical significance of therapeutic approach to treatment}

Furthermore, therapeutic assessment is based on the empirically supported psychological therapies. By this marker, I refer to those psychological treatments that have been exposed to assessment using the recognised methods of psychological science. Though much has been said and written about the significance of therapeutic assessment to treatment; some of the used terms such as empirically validated, empirically supported and empirically evaluated are still contestable in psychological research. For example, the first connotation means that a treatment has already been validated (Garfield, 1996), and proved effective. However, this does not mean the validation is completed and closed, and the therapeutic assessment does not produce a thorough success (Kendall, 1989). Also, the method of assessment is not resolved even if a number of studies offered supportive proof on it. The second expression means that the treatment has been supported with the condition that the backing comes from a suitable empirical study. The third idiom indicates that the treatments are empirically evaluated, that is, they have been empirically sustained. However, this is not unambiguous.

Another way of hypothesising this development is to look at the clients coming for treatment as part of a dysfunctional population and those who have completed as not part of that population anymore. This can be operationalised as follows: (a) The level of effectiveness resultant to treatment should fall outside the range of the dysfunctional population, where range is demarcated as ranging to two standard deviations beyond the mean for that population. (b) The level of effectiveness resultant to treatment should be within the level of normal population, that is, within two standard deviations of the mean of the population group. (c) The range of effectiveness subsequent to treatment should place client nearer to the mean of the functio- 
nal group than the dysfunctional group. This third meaning of the clinically significant change is the least illogical. This is because the definition was founded on a probability that scores would end in dysfunctional versus functional population distributions. However, clinically significant change is determined when a post-treatment score falls within the functional populace on the variable of interest. When this standard is met, it is statistically more probable to be drawn from the functional than the dysfunctional populace.

\section{Empirically documenting clinically significant change in therapeutic assessment}

In considering the determination of clinical significance of therapeutic assessment to treatment, evaluating the effectiveness of treatment can be done by the combination of objective empirical data and qualitative observational data. That is, clinicians should identify the strengths and feebleness of therapeutic interventions by using the art of science and not just a 'gut feeling'. This means that for treatment to bring about clinical significant change, the foundation of the therapy, particularly is philosophical orientation must be grounded on empirical science. Although the clinical philosophy that guides therapeutic assessment in clinical practice is complex as well as comprehensive, clinicians are expected to provide objective evaluations of the treatment effects. This is very essential because it impacts positively on the physical, intellectual and spiritual well-being of their clients as well as enhances their health and development in the mind, body and spirit.

Apart from this, empirical evaluations of treatment are essentially important because it offers the measurements of clinical significant change that are independent of the therapist's opinions. However, the main objections to this notion are mostly from the clinicians themselves. Research shows that most clinicians were of the opinion that 'data' are not required to tell them what works; they 'know' and that the treatments they offered are based on their professional knowledge ( Kipnis, 1994 ). While internal attributions for positive upshots are usually healthy, therapists are misled by taking the recognition for client improvements rather than controlling the alternate reasons of the outcomes (Kipnis, 1994). However, for an empirically supported therapeutic assessment, evidence should be resulting from the research clinics in addition to the initiator of the treatment.

Furthermore, a cursory scanning of the literature on therapeutic assessment revealed little on therapeutic values. However, it is astounding to know that limited organised empirical studies were carried out on the subject. This means that research on therapeutic approaches has not been comprehensively established and that they are not necessarily good. For instance, van der Kolk, (1996) maintained that as of 1996, there was only one research study on treatment of post-traumatic stress in children. However, empirically supported therapeutic assessment refers to as treatments that had been exposed to assessment using the accepted methods of psychological science. This means that the therapy has been supported with the condition that the support comes from an acceptable empirical study. That is, the evidence in question is empirical in nature. However, the existence of a research backing approach does not automatically imply that the approach is effective in a new contextual environment (generalisability). At the same time, lack of research does not imply that an approach is faulty, but rather shows that the method is yet to be fully confirmed. Also, an approach that worked for one group of people does not mean it would be effective for other diverse group.

Finn and Tonsager (1992) measured the clinical significant change in therapeutic assessment using the clients who participated in a short-term psychological assessment at the university counselling centre. Thirty-two clients participated and completed the Minnesota Multiphasic Personality Inventory-2 (MMPI-2; Butcher, Dahlstrom, Graham, Tellegen, \& Kaemmer, 1989) in the study and were given an hour feedback session using the shared method established by Finn (1996). Also, twenty-nine clients in a control group were examined and given the same level of therapeutic treatment (i.e., supportive nondirective psychotherapy) as alternative to a test feedback. Likened with the clients in the control group, those participants who took part in the MMPI-2 test indicated a substantial drop in symptomatic pain and an upsurge in their selfesteem both instantaneously after their feedback meeting and after 2 weeks. Similarly, the participants also showed a sign of confidence about their difficulties after the short-term assessment. 
Newman and Greenway (1997) sustained and duplicated the research conducted by Finn and Tonsager (1992) at Australian university counselling service and found that those clients who engaged in the brief assessment displayed high self-esteem and a decline in symptomatology after more than two follow-ups. Although the outcome sizes were fewer than those established by Finn and Tonsager (1992), the variations of those who participated in the assessment were clinically and statistically significant. Besides, the positive report from the clients' assessment was linked to the feedback given to them as well as the better quality of the design, and it shows that their actions are not related to their participation in the MMPI-2.

Also, a recent meta-analysis study conducted by Abbass, Kisely and Kroenke (2009) on short-term psychodynamic therapy for somatic disorders conducted on 23 studies involving 1870 patients suffering from a wide range of somatic conditions (e.g., dermatological, neurological, cardiovascular, respiratory, gastrointestinal, musculoskeletal, genitourinary, immunological) reported 0.69 and 0.59 for improvement in general psychiatric and somatic symptoms, respectively. However, among the studies on health care utilisation, it was found that $77.8 \%$ reductions in health care use were due to psychodynamic therapy.

Similarly, a meta-analysis examined the clinical significance of both the psychodynamic psychotherapy (14 studies) and CBT (11 studies) for personality disorders (Leichsenring \& Leibing, 2003) provide additional evidence of the effectiveness of therapeutic approach to treatment. The study as was reported in the American Journal of Psychiatry found that the mean length of treatment and the mean follow-up period between pre-treatment to post-treatment demonstrated the effectiveness of therapeutic assessment to treatment outcomes. In addition, a current review of short-term (average of 30.7 sessions) psychodynamic therapy for personality disorders (Messer \& Abbass, in press) reported effect sizes of 0.91 and 0.97 for general symptom and interpersonal functioning improvement, respectively. These meta-analyses typify the current and methodologically rigorous assessments that explained the clinical significant change in therapeutic assessment.

Additionally, Shedler-Westen Assessment Procedure (SWAP; Shedler \& Westen, 2007; Westen \& Shedler, 1999a) was used to assess the clinical significant change in therapeutic assessment. The methods sustained the clinical significance of therapeutic assessment by establishing the inner capacities and resources that evolved from the process. For example, SWAP measured a wide range of personality approaches, both healthy and pathological. The instrument demonstrated high reliability and validity relative to a wide range of criterion measures (Shedler \& Westen, 2007; Westen \& Shedler, 2007) and supported the clinical significance of therapeutic approach to mental health treatment (Westen \& Shedler, 1999a).

Though not much has been done on outcome studies that measure changes in inner capacities and resources using SWAP, the two research works on the issue raised a fascinating promise and suggested ways for future research of clinical significance in therapeutic assessment. One of these is a case study of a woman diagnosed with borderline personality disorder and assessed with the SWAP at the beginning of treatment and again after 2 years (Lingiardi, Shedler, \& Gazzillo, 2006). Apart from the significant reductions in SWAP scales that measure psychopathology, the patient's SWAP scores revealed the following effects: an increased capacity for compassion and greater sympathy to others' needs and emotional state; increased capability to identify other viewpoints even when feelings ran high; increased ability to ease and soothe herself; increased recognition and consciousness of the significance of her actions; increased ability to express herself orally; more precise and well-adjusted insights of people and situations; a greater capacity to appreciate humour and, possibly most essential, she had come to accept the throbbing past experiences and had found sense in them and developed from them. These outcomes indicated a significant change in client's condition by increased the score on the SWAP Healthy Functioning Index over the course of treatment, therefore, confirmed the clinical significance of therapeutic assessment to treatment.

\section{Discussion}

One of the intents of this paper was to provide an overview on the clinical significance of therapeutic approaches to treatment planning. This is important, particularly for readers who have not been open to therapeutic procedure or those who have not read it presented by a contemporary practitioner who used 
them for clinical practice. Another reason was to show the considerable empirical support that validates the clinical significance of therapeutic approach to treatment outcomes. In the course of writing this paper, I could not help being bumped by a number of ironies. One of these is that most scholars and practitioners who dismissed therapeutic approaches to psychological assessment in passionate tones; often do so in the name of science. Some champion a science of psychology based wholly in the experimental process, but forget the fact that the same experimental process produces results that support both the therapeutic ideas (e.g., Westen, 1998) and treatments. In light of the rise in empirical findings, blanket statements that therapeutic approaches lack scientific backing and cannot be clinically significant to treatment (e.g., Barlow \& Durand, 2005; Crews, 1996; Kihlstrom, 1999) are no longer defensible.

Second, it is also worth mentioning that relatively few clinicians are familiar with the research reviewed in this paper, particularly on the efficacy of therapeutic approach to treatment plans. Many clinical professionals and educators appear ill-prepared to react to challenge of clinical significant change from evidenceoriented contemporaries, students and policymakers despite the amassing of superior empirical evidence supporting the significance of therapeutic approach to treatment. Just as anti-assessment feeling may have obstructed spreading of the idea in academic environments, distrust of theoretical research methods may have hindered dissemination to psychotherapy groups (see Bornstein, 2001). Though such behaviour is now changing, nevertheless, this can only be gradual.

Third, the strength of clinical significance methodology is that it measured change in individual client level. Surprisingly, one of the empirical challenges in attaining significant change in therapeutic assessment is how to isolate and control variables. Most therapeutic approaches used multiple methods and this makes it hard to decide what change emanated from a particular component. This is true when considered a severe mental health service for a child and family system that used holistic and ecological approach. However, there were empirical researches that emphasised the components of treatment that are likely to be more effective than when the component is missing. Therefore, clinical psychologists should endeavour to accustom themselves with the principle and practice of clinical significant change in therapeutic assessment as this is relevant for gauging treatment outcomes in clinical practice.

Finally, scholars and academicians shared the blame for the poor state and the use of therapeutic approaches to treatment (Shedler, 2006b). Many researchers take for granted that clinicians are the intended users of clinical research (e.g., Task Force on Promotion and Dissemination of Psychological Procedures, 1995), but many of the psychoanalysis outcome studies and meta-analyses reviewed in this paper are obviously not carved for practitioners. If clinicians are indeed the intended 'users' of therapeutic assessment, then research on psychological assessment, particularly on clinical significant change must be user friendly (Westen, Novotny, \& Thompson-Brenner, 2005).

\section{Conclusions}

The debate about attaining clinical significant change in therapeutic assessment continues to take a centre stage in psychotherapy research. Though these processes are facing crucial challenges critical to their history, the problems will definitely reduce if clinicians and researchers embrace empirical treatment validity and work towards attaining clinical significant change in their dealing. The review of literature consistently and repeatedly shows the need for clients and therapist to attain the normal level of functioning like their counterparts at the end of treatment (Jacobson, Follette, \& Revenstorf, 1984; Jacobson et al., 1999; Jacobson \& Truax, 1991), and that the return to normal functioning should be used to define the clinical significance. This and other evidence shows why the debate on clinical significance moved beyond infrequent reference by a group of clairvoyant observers (e.g., Meyer et al., 2001) to a sprightly subject for argument and examination (Riddle et al., 2002). Based on this, this review is consistent with and brings up to date those of other reviews on clinical significant change in therapeutic assessment.

To sum up this review, therapeutic assessment is a strategy for improving treatment and it helps clinical professionals to achieve constructive and clinical significant change in clients (e.g., Finn, 2007). Methods such as the SWAP are relevant to treatment plans and can be integrated in future research as a process 
for gauging the clinical significant change in therapeutic assessment. Though methodological boundaries prevent drawing causal conclusions from the reviewed studies, nevertheless, it proposed that therapeutic assessment is not only used to ease symptoms, but also advance inherent capacities and resources that allow clients to reach clinical significant change or optimal treatment condition. Whether or not all clinicians use clinical significant change to measure therapeutic outcomes or researchers study them, it is clearly an intervention process that support people desired positive change and outcomes in their life. Perhaps this is why psychologists, irrespective of their own theoretical orientations, tend to choose the method for assessment in clinical practice (Norcross, 2005).

\section{Recommendation and future direction}

Research on therapeutic intervention in treatment planning is important. In fact, the subject has gone a long way to offer evidence that supports the clinical significant change in clients. This did not come out of the blue, but was part of the debate for a more effective and clinically supported therapeutic change in clinical practice. Though the proponent of clinical significance have long canvassing it's importance to treatment outcome, nonetheless there are still preponderance of assumptions based on minor numerical effects of little practical meaning and an inclination toward over construing group variances that are not beneficial to clients, but yet used by scientists to check their a priori hypotheses. Therefore, the future research should focus on the usage of clinical significant change as criteria to measure the effectiveness of therapeutic approach to treatment, particularly on clients' characteristics, treatment selection and outcomes. Based on this, the following recommendations were suggested:

1. Future research on psychological assessment should focus on information regarding clients and therapist demography and be more consumer relevant.

2. Psychology professional body should embrace professional development training that focus on therapeutic models. This will go a long way to effect client change and improve treatment processes.

3. Policy makers should re-examine and embrace proficiency standards and guidelines for psychological assessment practice that focus on basic features of therapeutic models.

4. Clinical psychologist should identify successful models of treatment decision making in light of patient preferences.

5. Finally, efforts should be channelled towards enabling training on ethics, proficiency and evidencebased practice in therapeutic assessment. This will go a long way to rectify the undesirable attitudes about psychological assessment in clinical practice.

\section{References}

Abbass, A., Kisely, S., \& Kroenke, K. (2009). Short-term psychodynamic psychotherapy for somatic disorders: Systematic review and metaanalysis of clinical trials. Psychotherapy and Psychosomatics, 78, 265- 274. doi:10.1159/000228247

Anastasi, A., \& Urbina, S. (1997). Psychological testing (7th ed.). Upper Saddle River, NJ: Prentice-Hall.

Barlow, D. H., \& Durand, V. M. (2005). Abnormal psychology: An integrative approach (4th ed.). Pacific Grove, CA: Brooks/Cole.

Bornstein, R. (2001). The impending death of psychoanalysis. Psychoanalytic Psychology, 18, 3-20. doi:10.1037/07369735.18.1.3

Butcher, J. N., Dahlstrom, W. G., Graham, J. R., Tellegen, A., \& Kaemmer, B. (1989). Minnesota Multiphasic Personality Inventory-2 (MMPI-2): Manual for administration and scoring. Minneapolis: University of Minnesota Press.

Crews, F. (1996). The verdict on Freud. Psychological Science, 7, 63-67.

Crosby, R. D., Kolotkin, R. L., \& Williams, G. R. (2003). Defining clinically meaningful change in health-related quality of life. Journal of Clinical Epidemiology, 56, 395-407. doi:10.1016/S0895- 4356(03)00044-1

De La Cour, A. T. (1986). Use of the focus in brief dynamic psychotherapy. Psychotherapy: Theory, Research, and Practice, 23, 133-139.

Eisman, E., Dies, R., Finn, S. E., Eyde, L. D., Kay, G. G., Kubiszyn, T. W., Meyer, G. J., \& Moreland, K. L. (2000). Problems and limitations in the use of psychological assessment in contemporary health care delivery. Professional Psychology: Research and Practice, 31, 131-140. 
Finn, S. E, \& Tonsager M.E.(1992). Therapeutic effects of providing MMPI-2 test feedback to college students awaiting therapy. Psychological Assessment.; 4,278-287.

Finn, S. E, \& Bunner, M. R. (1993). Impact of test feedback on psychiatric inpatients' satisfaction with assessment. Paper presented at the 28th Annual Symposium on Recent Developments in the Use of the MMPI, St. Petersburg Beach, FL

Finn, S. E. (1996). Manual for using the MMPI-2 as a therapeutic intervention. Minneapolis, MN: University of Minnesota Press.

Finn, S. E. (2007). In our clients' shoes: Theory and techniques of therapeutic assessment. Mahwah, NJ: Erlbaum

Garfield, S. L. (1996). Some problems with “validated” forms of psychotherapy.( Clinical Psychology: Science and Practice, 3 , 218-229.)

Goldman, L. (1972). Tests and counseling: The marriage that failed. Measurement and Evaluation in Guidance, 4, 213-220.

Jacobson, N. S., Roberts, L. J., Berns, S. B., \& McGlinchey, J. B. (1999). Methods for defining and determining the clinical significance of treatment effects: Description, application, and alternatives. Journal of Consulting and Clinical Psychology, 67, 300-307. doi:10.1037/0022-006X.67.3.300

Jacobson, N. S., \& Truax, P. (1991). Clinical significance: A statistical approach to defining meaningful change in psychotherapy research. Journal of Consulting and Clinical Psychology, 59, 12-19. doi:10.1037/ 0022-006X.59.1.12

Jacobson, N. S., Follette, W. C., \& Revenstorf, D. (1984). Psychotherapy outcome research: Methods for reporting variability and evaluating clinical significance. Behavior Therapy. 15, 336-352.

Kazdin, A. E. (2001). Almost clinically significant ( $\left.p_{-} .10\right)$ : Current measures may only approach clinical significance. Clinical Psychology: Science and Practice, 8, 455-462. doi:10.1093/clipsy/8.4.455

Kazdin, A. E., \& Wilson, G. T. (1978). Evaluation of behavior therapy: Issues, evidence, and research strategies. Cambridge, MA: Ballinger.

Kendall, P. C. (1989). The generalization and maintenance of behavior change: Comments, considerations, and the "no-cure" criticism. ( Behavior Therapy, 20, 357-364.)

Kihlstrom, J. F. (1999). A tumbling ground for whimsies? Contemporary Psychology, 44, 376-378. doi:10.1037/002604

Kipnis, D. (1994). Accounting for the use of behavior technologies in social psychology (American Psychologist, 49, 165-172.)

Leichsenring, F., \& Leibing, E. (2003). The effectiveness of psychodynamic therapy and cognitive behavior therapy in the treatment of personality disorders: A meta-analysis. American Journal of Psychiatry,160, 1223-1232

Lingiardi, V., Shedler, J., \& Gazzillo, F. (2006). Assessing personality change in psychotherapy with the SWAP-200: A case study. Journal of Personality Assessment,

Meyer, G. J., Finn, S. E., Eyde, L. D., Kay, G. G., Moreland, K. L., Dies, R. R., Reed, G. M. (2001). Psychological testing and psychological assessment. American Psychologist, 56, 128-165.

Newman, M. L., \& Greenway, P. (1997). Therapeutic effects of providing MMPI-2 test feedback to clients at a university counseling service: A collaborative approach. Psychological Assessment, 9, 122-131.

Nietzel, M. T, \& Trull, T. J. (1988). Meta-analytic approaches to social comparisons: A method for measuring clinical significance. Behavioral Assessment, 10, 146-159.

Norcross, J. C., Karpiak, C. P., \& Santoro, S. O. (2005). Clinical psychologists across the years: The Division of Clinical Psychology from 1960 to 2003. Journal of Clinical Psychology, 61, 1467-1483.

Ogles, B. M., Lunnen, K. M., \& Bonesteel, K. (2001). Clinical significance: History, application, and current practice. Clinical Psychology Review, 21, 421-446.

Riddle, B. C., Byers, C. C., \& Grimesey, J. L. (2002). Literature review of research and practice in collaborative assessment. Humanistic Psychologist,30, 33-48.

Rogers, C. R. (1951). Client-centered therapy. Boston: Houghton Mifflin.

Roth, A., Fonagy, P. (2005). What Works for Whom? A Critical Review of Psychotherapy research, 2nd edn. Guilford Press : New York.

Shedler, J. (2006b). Why the scientist-practitioner schism won't go away. The General Psychologist, 41(2), 9-10. Retrieved from http:// ww.apa.org/divisions/div1/archive.html

Shedler, J., \& Westen, D. (2007). The Shedler-Westen Assessment Procedure (SWAP): Making personality diagnosis clinically meaningful. Journal of Personality Assessment, 89, 41-55.

Task Force on Promotion and Dissemination of Psychological Procedures. (1995). Training in and dissemination of empiricallyvalidated treatments: Report and recommendations. The Clinical Psychologist, 48(1), 3-23

Tharinger, D. J., Finn, S. E., Wilkinson, A. D., \& Schaber, P. M. (2007). Therapeutic assessment with a child as a family intervention: A clinical and research case study.

Thompson, B. (2002). "Statistical," "practical," and "clinical”: How many kinds of significance do counselors need to consider? Journal of Counseling \& Development, 80, 64-71. doi:10.1002/j.1556-6678.2002.tb00167.x

van der Kolk, B.A., McFarlane, A.C. \& Weisaeth, L. (Eds.) (1996). Traumatic Stress, the Effects of Overwhelming Experience on Mind, Body, and Society. New York: Guilford Press.

Westen, D. (1998). The scientific legacy of Sigmund Freud: Toward a psychodynamically informed psychological science. Psychological Bulletin, 124, 333-371. doi:10.1037/0033-2909.124.3.333

Westen, D., \& Shedler, J. (1999a). Revising and assessing Axis II, Part 1: Developing a clinically and empirically valid assessment method. American Journal of Psychiatry, 156, 258-272. 
Westen, D., Novotny, C. M., \& Thompson-Brenner, H. (2005). EBP _ EST: Reply to Crits-Christoph et al. (2005). and Weisz et al. (2005).Psychological Bulletin, 131, 427-433. doi:10.1037/0033-2909.131.3.427

Westen, D., \& Shedler, J. (2007). Personality diagnosis with the Shedler- Westen Assessment Procedure (SWAP): Integrating clinical and statistical measurement and prediction. Journal of Abnormal Psychology,116, 810-822. doi:10.1037/0021843X.116.4.810

Wolf, M. M. (1978). Social validity: The case for subjective measurement or how applied behavior analysis is finding its heart. Journal of Applied Behavior Analysis. 11. 203- 214 